\title{
Uveitis presenting with iris bombe in a patient with HIV: the importance of multi-disciplinary management - a case study
}

\author{
Thomas RP Taylor ${ }^{1}$, Neil Shah', Rashmi Akshikar ${ }^{2}$ \\ ${ }^{1}$ Barts Health NHS Trust, Academic Foundation Program, Royal London Hospital, London, United Kingdom \\ ${ }^{2}$ Imperial College Healthcare NHS Trust, London, United Kingdom
}

\begin{abstract}
This case study concerns a 53-year-old female presenting to an eye-hospital in London with uveitis, on a background of well-controlled HIV. After investigation, no cause for the uveitis was found other than the HIV itself, and the patient was treated with immunosuppressants. Because of the nature of her underlying HIV infection, help was sought from HIV specialists, who gave advice on the management, including alteration of corticosteroid dosing due to the risk of drug-interactions between her uveitis and HIV treatments. Of particular concern was the risk of cytochrome P450 3A inhibition by cobicistat and the potential for iatrogenic Cushing syndrome through elevated steroid doses. This case is used as an example to highlight the importance of the multi-disciplinary team in the management of complex conditions, as well as to add to the literature in support of the HIV virus itself as a cause of uveitis.
\end{abstract}

KEY WORDS: uveitis; HIV; iris bombe; multi-disciplinary; inflammation

Ophthalmol J 2020; Vol. 5, 29-32

\section{CASE PRESENTATION}

A 53-year-old Afro-Caribbean female with a background of well-controlled human immunodeficiency virus (HIV) suffered subacute onset left sided ocular pain and loss of vision.

One year prior to presentation, she had a suspected reactivation of ocular (retinal) toxoplasmosis treated appropriately. Diagnosed over 25 years ago, her HIV was known to be triple-class antiretroviral drug resistant (TC-DR). Despite extensive antiretroviral therapy changes, she was well controlled on Symtuza (combination of darunavir, cobicistat, emtricitabine, and tenofovir alafenamide) at the time of presentation. Her most recent CD4 count was 840 cells $/ \mathrm{mm}^{3}$ with an undetectable HIV-1 viral load.
At presentation with a five-day history of worsening ocular pain, floaters, and loss of vision, her left intraocular pressure (IOP) was $42 \mathrm{mmHg}$, and her visual acuity was $\mathrm{CF}$ in the affected eye. Extensive corneal oedema was present (Fig. 1) with iris bombe demonstrated in three quadrants as well as a large crystalline lens on B-scan ocular ultrasound. With no clinical fundal view, B-scan also showed a retinal traction band expanding temporally with no retinal detachment. A diagnosis of hypertensive uveitis and iris bombe was made, with particular note of the immunocompromised state.

She had no other systemic symptoms. In particular, she had no weight loss, fever, night sweats, rashes, or cough, which could point to an aetiology. There was no history of, or high-risk contact with, 


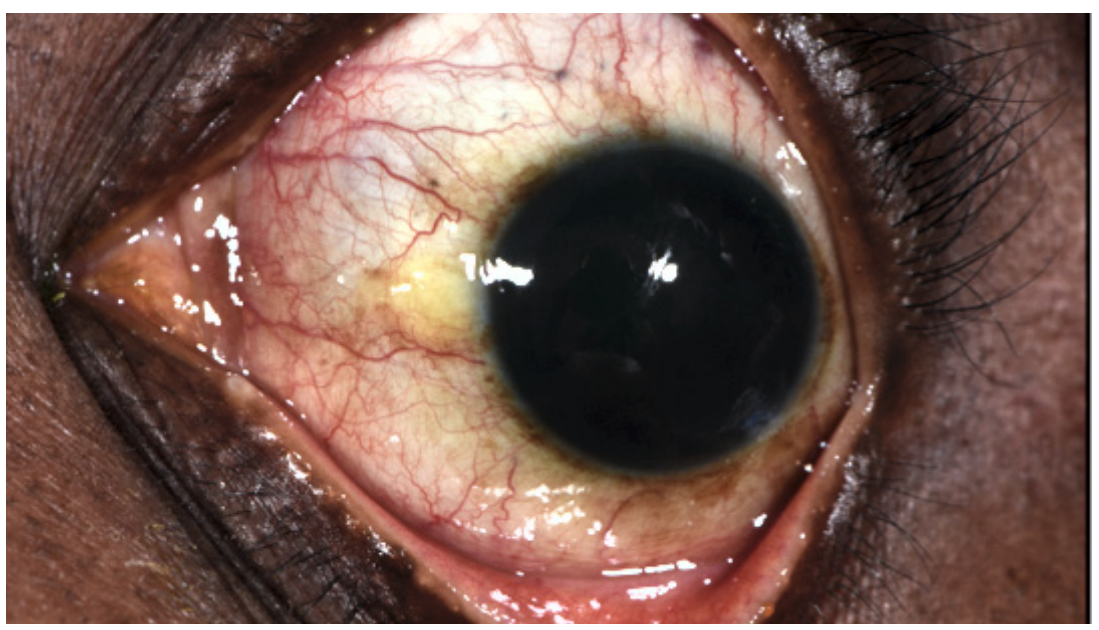

FIGURE 1. Affected eye showing significant corneal oedema

mycobacterium tuberculosis (TB). Extra-ocular clinical examination was within normal limits, with no lymphadenopathy.

Intraocular pressure lowering was immediately attempted with intravenous acetazolamide. Topical carbonic anhydrase inhibitor, beta blockers, anti-muscarinic, prostaglandin analogues, and alpha agonists [dorzolamide and timolol (Cosopt), atropine, latanoprost and iopidine, respectively] were also administered to aid this. Oral and topical steroids were started to treat the uveitis, the underlying cause of the raised IOP and loss of vision. As she was on a protease inhibitor, this was all in close discussion with the HIV team and specialist pharmacists, to prevent adverse effects on HIV control. Anti-emetics, analgesics, and benzodiazepines were also prescribed for symptom relief.

Over three days, her ocular pain settled, intraocular pressure reduced to $10 \mathrm{~mm} \mathrm{Hg}$, and she was discharged with a reducing regime of oral prednisolone as well as a topical steroid and non-steroidal anti-inflammatory drops as a steroid-sparing alternative. Unfortunately, her visual acuity remained $\mathrm{CF}$ in the left eye despite treatment.

In the following two months, her IOP became persistently high at $34 \mathrm{~mm} \mathrm{Hg}$ despite oral acetazolamide. An emergency micropulse diode laser trabeculoplasty (MDLT), using laser to alter the trabecular network and aid flow of the aqueous humour and thus reduce IOP, was successfully performed. Due to persistent inflammation, a dense cataract has formed. She now awaits a surgical iridectomy and cataract surgery with intravitreal steroid to open up the iridocorneal angle further and improve visual acuity.
Despite extensive testing, no cause of the aetiology of her uveitis was found. Her repeated HIV-1 viral load was less than 20 and CD4 count 373 cells $/ \mathrm{mm}^{3}$.

Throughout her treatment, the HIV team were very closely involved. Whilst on immunosuppressive steroids, it was decided to intensify her current HIV therapy with the addition of dolutegravir, an integrase inhibitor. She remains systemically well from an HIV perspective at present, and ongoing collaborative care remains essential because long-term immunosuppression for ocular pathology is likely.

\section{DISCUSSION}

This patient presented with uveitis (inflammation of the iris, ciliary body, and choroid) leading to raised IOP after developing iris bombe. This is where inflammation causes the iris to become adherent to the crystalline lens, preventing aqueous humour outflow. Raised IOP is dangerous due to its potential to cause irreversible optic neuropathy (glaucoma).

Uveitis is associated with many conditions including autoinflammatory disorders, infectious diseases, or it may be idiopathic. HIV is a disease commonly associated with uveitis, and it is estimated that $70-80 \%$ of patients with untreated HIV will develop an ocular condition at some point, of which half will be inflammation or uveitis [1].

Uveitis and other ocular complications of HIV are typically caused by opportunistic infections such as tuberculosis, syphilis, and toxoplasmosis, as well as malignant processes such as lymphoma secondary to progressive immune dysfunction. However, there 
are also reports of uveitis occurring in HIV-infected patients without any suspected causative agents other than the HIV virus itself $[2,3]$. This implicates that the eye itself could be a sanctuary for HIV.

Certain drugs used to treat HIV are also associated with a risk of uveitis, particularly medications containing rifabutin [4] or cidofovir [5]. Symtuza, however, is not associated with this risk. Only one published study has found a weak link between a similar class of medication containing three of the four constituent drugs and uveitis. In all cases, the uveitis resolved without stopping the drug [6]. Given no evidence to the contrary, it is likely that the HIV infection itself was the causative organism in this case, although intraocular fluid was not sent for culture to confirm this.

Anterior uveitis in HIV tends to be asymptomatic [7]. A case series of symptomatic uveitis in HIV found four other patients over a four-year period in one hospital with CD4 counts over $200 \mathrm{cells} / \mathrm{mm}^{3}$ and symptomatic uveitis with no concurrent illness [7]. Other symptomatic cases, which were associated with other aetiologies, such as tuberculosis and herpes zoster, all had CD4 counts $<200$ cells $/ \mathrm{mm}^{3}$.

This case highlights the comparative rarity of a patient with well-controlled HIV developing a symptomatic uveitis without clear underlying aetiology, as well as the importance of cooperation between ophthalmic and infectious disease specialists in the treatment of this condition.

The mainstay of treatment of non-infective uveitis is corticosteroids, which can be given via several routes, commonly topical and oral [8]. They need slow tapering over a period of weeks so as not to cause rebound inflammation when they are withdrawn. Drugs to paralyse the ciliary body are also given to relieve pain and prevent adhesions. If an infective cause is suspected, an appropriate antimicrobial is also added.

Despite the theoretical concerns over the use of immunosuppressive drugs such as corticosteroids in patients who are already immunosuppressed, studies have shown that short-term steroid use in HIV-positive individuals is relatively safe, well tolerated, and not associated with effects on HIV-1 RNA levels or CD4 cell counts $[9,10]$. Corticosteroid use is generally only a concern when high doses are used, or when the CD4 count is very low [11].

The issue with co-prescribing corticosteroids in patients being treated for HIV is not therefore a risk of worsening HIV control; instead, care must be taken due to drug-drug interactions between corticosteroids and HIV treatments. Cobisistat, one of the components of Symtuza, acts as a boosting agent for the effects of the protease inhibitor darunavir. Its main mechanism of action is inhibition of the enzyme cytochrome P450 3A (CYP3A), the liver enzyme that breaks down darunavir [12]. Inhibition of this enzyme therefore leads to increased levels and prolonged effects of darunavir. Many other drugs are also metabolised by CYP3A, the most relevant of which in this case are corticosteroids. Through the same mechanism of action, Cobisistat therefore increases plasma concentrations of corticosteroids given as treatment for uveitis and could risk development of steroid-associated side effects. This includes Cushing syndrome and secondary adrenal suppression through the hypothalamic-pituitary-adrenal axis.

There has been a reported case of this occurring in a patient on a CYP3A inhibitor and corticosteroid eye drops, which led to serious complications including avascular necrosis of the hip [13]. Conversely, there is also a theoretical risk that the steroid (dexamethasone) eye drops could alter the efficacy of the protease inhibitor darunavir. This is because dexamethasone can act as an inducer of the CYP3A enzyme, of which darunavir is a substrate, which could worsen HIV control [14]. Accordingly, care must be taken when co-prescribing these medications because they have the potential to cause serious, irreversible side-effects as well as the worsening of underlying HIV control. Specialists must thus be involved to ensure safe prescribing.

In our patient, the dose of oral corticosteroids was reduced by a third to compensate for the increased systemic exposure. Punctal occlusion was also taught to minimise systemic absorption of topical dexamethasone drops. The decision was also made to intensify HIV treatment with the addition of dolutegravir to counteract the potential reduction in efficacy through the induction of darunavir. Dolutegravir is not primarily metabolised by CYP3A and is therefore not subject to the same drug interactions discussed here [15].

Without input from multi-disciplinary specialists, this patient could have been at greater risk of serious iatrogenic complications.

\section{CONCLUSION}

This case showcases that ocular inflammatory emergencies, such as iris bombe, can present in the 
absence of obvious causal aetiology, and it adds to the literature in support of the HIV-1 virus itself as a cause of uveitis. Multi-disciplinary teamwork is key in the management of complex uveitis, especially with immunosuppressed individuals on medications pre-disposing to dangerous drug-drug interactions.

\section{Statement of competing interests}

The authors report no competing interests.

\section{REFERENCES}

1. Cunningham ET, Margolis TP. Ocular manifestations of HIV infection. N Engl J Med. 1998; 339(4): 236-244, doi: 10.1056/ NEJM199807233390406, indexed in Pubmed: 9673303.

2. Rothova A, Schneider M, de Groot-Mijnes JDF. Human immunodeficiency virus-induced uveitis: intraocular and plasma human immunodeficiency virus-1 RNA loads. Ophthalmology. 2008; 115(11): 2062-2064, doi: 10.1016/j.ophtha.2008.04.034, indexed in Pubmed: 18562005 .

3. Rosberger D, Heinemann MH, Friedberg D, et al. Uveitis associated with human immunodeficiency virus infection. Am J Ophthalmol. 1998; 125(3): 301-305, doi: 10.1016/s0002-9394(99)80135-4, indexed in Pubmed: 9512146.

4. Nichols C. Mycobacterium avium Complex Infection, Rifabutin, and Uveitis - Is There a Connection? Clin Infect Dis. 1996; 22(Suppl 1): S43-S49, doi: 10.1093/clinids/22.supplement_1.s43, indexed in Pubmed: 8785256.

5. Davis JL, Taskintuna I, Freeman WR, et al. Iritis and hypotony after treatment with intravenous cidofovir for cytomegalovirus retinitis. Arch Ophthalmol. 1997; 115(6): 733-737, doi: 10.1001/ archopht.1997.01100150735008, indexed in Pubmed: 9194724.
6. Gaur A, Kizito H, Prasitsueubsai W, et al. Safety, efficacy, and pharmacokinetics of a single-tablet regimen containing elvitegravir, cobicistat, emtricitabine, and tenofovir alafenamide in treatment-naive, HIV-infected adolescents: a single-arm, open-label trial. Lancet HIV. 2016; 3(12): e561-e568, doi: 10.1016/s2352-3018(16)30121-7, indexed in Pubmed: 27765666.

7. Verma S, Hughes JD, Mabey D, et al. Symptomatic anterior uveitis in HIV-positive patients. Int J STD AIDS. 1999; 10(4): 268-274, doi: 10.1258/0956462991913925, indexed in Pubmed: 12035782.

8. Uveitis - NICE CKS. Cks.nice.org.uk. (2019) . https://cks.nice.org.uk/ uveitis\#!backgroundsub:1.

9. McComsey GA, Whalen CC, Mawhorter SD, et al. Placebo-controlled trial of prednisone in advanced HIV-1 infection. AIDS. 2001; 15(3): 321-327, doi: 10.1097/00002030-200102160-00004, indexed in Pubmed: 11273211.

10. Andrieu JM, Lu W, Levy R. Sustained Increases In Cd4 Cell Counts In Asymptomatic Human. J Infect Dis. 1995; 171(3): 523-530, doi: 10.1093/infdis/171.3.523, indexed in Pubmed: 7876597 .

11. Nelson MR, Erskine D, Hawkins DA, et al. Treatment with corticosteroids - a risk factor for the development of clinical cytomegalovirus disease in AIDS. AIDS. 1993; 7(3): 375-378, doi: 10.1097/00002030199303000-00011, indexed in Pubmed: 8097096.

12. Tseng A, Foisy M. Important Drug-Drug Interactions in HIV-Infected Persons on Antiretroviral Therapy: An Update on New Interactions Between HIV and Non-HIV Drugs. Curr Infect Dis Rep. 2012; 14(1): 67-82, doi: 10.1007/s11908-011-0229-1, indexed in Pubmed: 22125049.

13. Molloy A, Matheson NJ, Meyer PAR, et al. Cushing's syndrome and adrenal axis suppression in a patient treated with ritonavir and corticosteroid eye drops. AIDS. 2011; 25(10): 1337-1339, doi: 10.1097/ QAD.0b013e328347c09c, indexed in Pubmed: 21659797.

14. HIV insite (2019). Interactions with Dexamethasone. http://hivinsite. ucsf.edu/insite?page $=$ ar-00-02\&post $=8 \pi \mathrm{m}=138$.

15. Castellino S, Moss L, Wagner D, et al. Metabolism, excretion, and mass balance of the HIV-1 integrase inhibitor dolutegravir in humans. Antimicrob Agents Chemother. 2013; 57(8): 3536-3546, doi: 10.1128/ AAC.00292-13, indexed in Pubmed: 23669385. 